\title{
Templated assembly of albumin-based nanoparticles for simultaneous gene silencing and magnetic resonance imaging
}

Received 00th January 2012, Accepted 00th January 2012

DOI: $10.1039 / \times 0 \times x 00000 x$

\author{
Damien Mertz, ${ }^{\text {a,b,c }}$ Christine Affolter-Zbaraszczuk, ${ }^{\text {b,d }}$ Julien Barthès, ${ }^{b}$ Jiwei Cui, ${ }^{\text {e }}$ \\ Frank Caruso, ${ }^{\text {e }}$ Thomas F. Baumert, ${ }^{\mathrm{c}, \mathrm{f}}$ Jean-Claude Voegel, ${ }^{\mathrm{b}, \mathrm{c}}$ Joelle Ogier, ${ }^{\mathrm{b}, \mathrm{c}, \mathrm{d}}$ \\ Florent Meyer ${ }^{\text {b,c,d }}$
}

www.rsc.org/

In this article, we address the design of innovative human serum albumin (HSA)-based nanoparticles loaded with silencing RNA and grafted with gadolinium complexes. The non-covalent siRNA/HSA assembly is formed on isobutyramide-modified mesoporous silica and the selfsupported HSA-based nanoparticles (ca. $100 \mathrm{~nm}$ size) are obtained following the silica templates dissolution. These original protein particles provide simultaneous magnetic resonance imaging contrast enhancement and cellular in vitro gene silencing.

The design of "theranostic" nanoplatforms that provide simultaneous drug delivery and clinical imaging has become a rapidly developing field. ${ }^{1}$ Such nanosystems encompass the development of integrated systems that incorporate multiple functionalities, such as tumor cell targeting, high resolution/sensitivity imaging, and drug release at a desired biological site. These materials are envisioned, for instance, for image-guided delivery of anticancer drugs (e.g., chemotherapeutics or photo/hyperthermal agents) that can be monitored by magnetic resonance imaging (MRI), computed tomography (CT), or positron emission tomography (PET). ${ }^{2}$ Among the range of existing antitumor therapeutics, silencing RNA (siRNA) has emerged as a new powerful therapeutic tool to address the challenges of tumor heterogeneity. ${ }^{3}$ The broad potential of RNA therapeutics to regulate the expression of a range of genes is expected to impact the future of nanomedicine. ${ }^{4}$ However, key limitations for clinical application of siRNA-based approaches remain, including low efficiency for the delivery of naked siRNA and its in vivo enzymatic degradation by nucleases after injection. Targeted delivery mediated by nanovectors offer advantages to considerably increase the efficacy of the treatment by reducing both the dose and the toxicity of the delivered molecule. Nanoparticles targeting capability of overexpressed receptors in cancer cells is generally afforded by the grafting of specific ligands ${ }^{5}$ including sugars, peptides, or antibodies that can be bound to the nanoparticle surface through various chemistries (e.g., electrostatic interactions, carbodiimide or click chemistries). Among nanovectors, lipid vesicles or polymer assemblies have been widely used as nanovectors for siRNA delivery. ${ }^{6}$ The ability of liposomes to deliver their contents (e.g., drugs, proteins or nucleic acids) has made these nanoparticles attractive for various applications. Cationic polymeric nanocarriers, such as polyethyleneimine (PEI)-based nanocarriers, offer several advantages, including high transfection efficacy and endosomal escape. Polymer capsules with nanometer-sized thin walls have also emerged as nanovehicles for drug delivery application. ${ }^{7}$ Recently, Becker et al. ${ }^{8}$ reported the encapsulation and delivery of siRNA using microcapsules composed of thiolated poly(methacrylic acid) (PMASH) to target prostate cancer cells. However, issues present with some of these nanovectors are liposome fragility reported in vivo, induction of cell necrosis, and apoptosis with the use of PEI and risks at short or mid-term of cytotoxicity or immunogenicity associated with the use of synthetic polymers. Thus, one crucial challenge in the field of carrier-based drug delivery remains the development of safe, robust and efficient nanovectors made of biological macromolecules, which can be rapidly removed or metabolized by the organism. There are few studies that report the design and application of biopolymer-based nanocarriers. Further, the combination of siRNA and imaging agents within a unique formulation of biomacromolecules is still in its infancy and it concerns essentially assemblies coated at the surface of inorganic nanoparticles. ${ }^{9}$

Herein, we address the design of human serum albumin (HSA)based nanoparticles for simultaneous gene silencing and MRI applications. HSA, which is the most abundant plasma protein, has emerged as a promising building-block for nanovectors because of its non-toxicity, non-immunogenicity, biodegradability, and propensity for high circulation time in the blood. ${ }^{10}$ Recently, we reported an efficient method based on the use of isobutyramide (IBAM) grafts and its derivatives to assemble various biomacromolecules, including proteins, at interfaces or freestanding. ${ }^{11}$ This approach has the advantage of a simplified procedure for the design of macromolecular particles, especially those made of biological polymers, without the need for further cross-linking or the addition of adjuvants. In the current study, we translate this approach to design original and efficient HSA-based nanoparticles of $c a .100 \mathrm{~nm}$ size loaded with siRNA and grafted with macrocyclic gadolinium complexes (DOTA-Gd). These DOTA-Gd complexes have similar structures to the well-established MRI contrast agents approved for clinical use, Dotarem ${ }^{\mathrm{TM}}$ or Gadovist ${ }^{\mathrm{TM}}$. These nanoparticles are obtained through the recently developed mesoporous silica (MS) templating method ${ }^{11,12}$ (Figure 1). The biomacromolecular architecture of siRNA and HSA is achieved by 
the use of IBAM binders grafted at the MS template surface, which promote the non-covalent biomacromolecular assembly within the MS pores. Following subsequent covalent attachment of the DOTAGd complexes and after template dissolution, Gd-modified siRNA/HSA replicated nanoparticles (denoted RNPs) are formed.

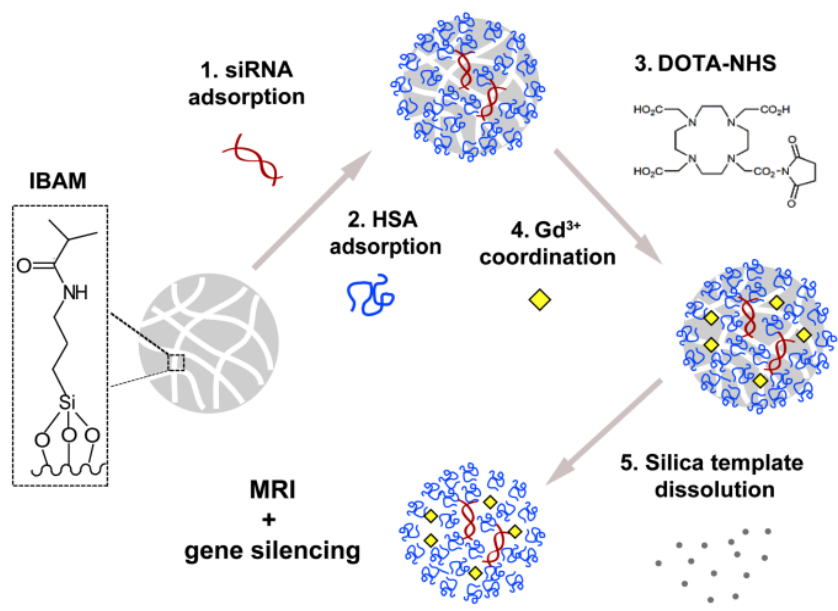

Figure 1. Scheme of the process to form Gd-modified siRNA/HSA RNPs. siRNA and HSA are non-covalently assembled by their successive adsorption onto IBAM-modified MS particles. Then DOTA-Gd complexes are covalently attached to the amino reactive parts of the RNPs through coupling reaction with DOTA-NHS macrocyclic ligand followed by chelation with $\mathrm{Gd}^{3+}$. Removal of the underlying silica template leads to stable Gd-modified siRNA/HSA RNPs.

The design of the RNPs depends directly on the synthesis of the MS acting as sacrificial templates. MS nanoparticles with an average diameter of $\sim 200 \mathrm{~nm}$ (2-4 $\mathrm{nm}$ and 20-40 nm porosity) were synthesized according to a modified literature method. ${ }^{13}$ The transmission electronic microscopy image (Figure 2A) shows homogenously formed MS of controlled size. The internal porosities of the MS were modified with IBAM grafts according to the method reported described previously. ${ }^{11}$ The biomacromolecular assembly of siRNA/HSA was formed within IBAM-modified MS particles through successive physical adsorption of these two biopolymers in aqueous solution ( $2 \mathrm{~h}$ respectively for siRNA and then $2 \mathrm{~h}$ for HSA). To quantify the amount of siRNA loaded in the MS particles as a function of the siRNA concentration, we investigated the adsorption of fluorescein isothiocyanate labeled siRNA (siRNA ${ }^{\text {FITC }}$ ) within the IBAM-modified MS particles by fluorescence spectroscopy analysis of the supernatant (see calibration curve of siRNA ${ }^{\text {FITC }}$ in Figure $\mathrm{S} 1 \mathrm{~A})$. Figure 2B shows the evolution of the siRNA quantity adsorbed ( $\mu \mathrm{g} . \mathrm{mg}^{-1}$ of $\mathrm{MS}$ ) relative to the concentration of siRNA $\left(\mu \mathrm{g} . \mathrm{mL}^{-1}\right)$ initially incubated in solution. These results show that the siRNA amounts adsorbed onto IBAM-MS can be tuned according to a linear profile from 6 to $50 \mu \mathrm{g}$ of siRNA per mg of MS after incubation with siRNA solutions of concentrations ranging from 25 to $200 \mu \mathrm{g} . \mathrm{mL}^{-1}$. Spectroscopy analysis of the supernatant shows that in this concentration range, all of the siRNA initially incubated is fully adsorbed within the MS silica, indicating that still numerous IBAM sites remain available for further adsorption. FITC-labeled HSA (HSA ${ }^{\text {FITC }}$ ) with variable concentrations from 0.03 to 4 mg. $\mathrm{mL}^{-1}$ was thus subsequently co-adsorbed on IBAM-modified MS coated with $20 \mu \mathrm{g}$ of siRNA per mg of MS. [HSA ${ }^{\mathrm{FITC}}$ ] concentration in the supernatant was measured after $2 \mathrm{~h}$ incubation and RNPs' centrifugation (see calibration curve of $\mathrm{HSA}^{\text {FITC }}$ in Figure S1B).
Figure 2C shows the resulting adsorption curve of $\mathrm{HSA}^{\mathrm{FITC}}$, which reaches an adsorbed amount of $358 \pm 5 \mu \mathrm{g}$ of $\mathrm{HSA}^{\text {FITC }} \mathrm{mg}^{-1}$ MS after incubation of $\mathrm{HSA}^{\mathrm{FITC}}$ solution at $4 \mathrm{mg} \mathrm{mL}^{-1}$. Finally, this method of co-adsorption, first of siRNA and then of HSA, allows the formation of IBAM-siRNA-HSA coatings within the MS, with tunable quantities of siRNA and of HSA.
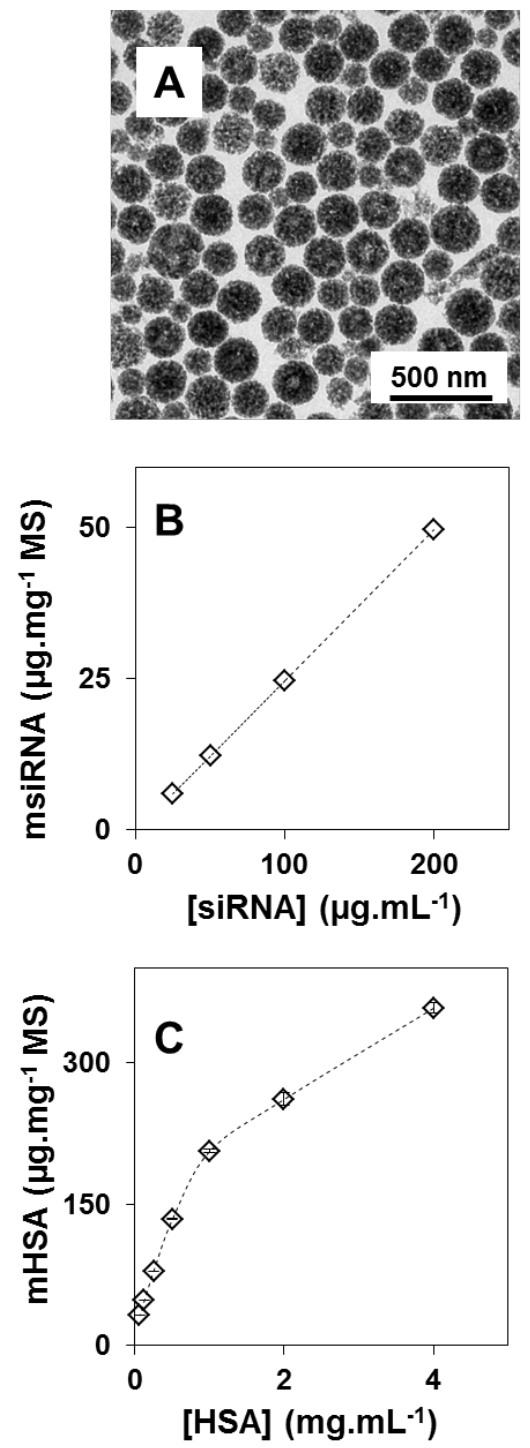

Figure 2. Assembly of siRNA/HSA coatings onto IBAM-modified MS. (A) TEM image of the MS sacrificial templates. Adsorption curves performed by spectrofluorimetry analysis of (B) the adsorbed amount of siRNA ${ }^{\text {FITC }}$ onto IBAM-modified MS versus the [siRNA] initially in solution and of (C) the adsorbed amount of HSA ${ }^{\text {FITC }}$ onto siRNA-coated (20 $\mu \mathrm{g} \cdot \mathrm{mg}^{-1}$ MS) IBAM-modified MS versus the [HSA] initially in solution.

With the aim to afford MRI properties to the biomacromolecular assemblies, Gd-DOTA complexes were covalently attached to the amine groups $\left(\mathrm{NH}_{2}\right.$, from lysine and arginine residues) of the adsorbed HSA via a coupling reaction using an excess of DOTA ligand (stoichiometry DOTA: $\mathrm{NH}_{2}$ ranging from 1:1 to 10:1), followed by chelation of $\mathrm{Gd}^{3+}$ cations (stoichiometry Gd:DOTA, 20:1 at room temperature). Four different formulations of RNP nanospheres were prepared with various amounts of siRNA and 
HSA: $10 / 62,40 / 62,10 / 206$ and $10 / 357 \mu g$ of siRNA/HSA adsorbed.mg ${ }^{-1}$ MS (denoted as si10-hsa62, si40-hsa62 si10-hsa206 and si10-hsa357). Si10-hsa62 and si40-hsa62 RNPs were grafted with Gd complexes at a ratio DOTA: $\mathrm{NH}_{2}$ of 1:1 while si10-hsa206 and si10-hsa357 RNPs were grafted at a ratio of DOTA: $\mathrm{NH}_{2}$ 10:1. Homogenous colloidal dispersions of RNPs were observed for the three samples after removal of the underlying silica template with 2 $\mathrm{M} \mathrm{HF} / 8 \mathrm{M} \mathrm{NH}_{4} \mathrm{~F}$ (buffered solution at $\mathrm{pH}$ 5), three washing steps and redispersion with Milli-Q water. Diffusion light scattering (DLS) analysis of si10-hsa62 and si10-hsa357 RNPs in water indicated respectively a hydrodynamic diameter respectively of $44 \pm$ $8 \mathrm{~nm}$ and $164 \pm \ldots \mathrm{nm}$ (Figure 3.A). TEM imaging performed in dried conditions confirmed the DLS values for these two types of RNPs with: $34 \pm 3 \mathrm{~nm}$ and $140 \pm 34 \mathrm{~nm}$ (Figure 3.B and C respectively). Confocal microscopy images of RNP dispersions prepared with siRNA ${ }^{\text {Red }}$ and HSA $^{\text {FITC }}$ (si10-hsa50) indicated the presence of fluorescence from both of the biopolymers within the RNPs (Figure S2). These RNPs were imaged by atomic force microscopy (AFM) in the dried state (Figure 3D-F). AFM images show, for the three samples (si10-hsa62, si40-hsa62 and si10hsa206), homogenously stabilized and dispersed Gd-modified siRNA/HSA RNPs of size $57 \pm 13,82 \pm 22$, and $116 \pm 13 \mathrm{~nm}$, respectively, which corresponds to respective shrinking of 71,59 , and $42 \%$ of the initial template size. The shrinking measured can be attributed to the loss of silica after template removal and subsequent protein matter reorganization. The precise values of shrinking depend on the amount of macromolecules adsorbed and show that the more biopolymers are adsorbed, the less the shrinking effect is. Further, AFM z-profile analysis (Figure S3) allows estimation of the thickness of the particles (flattened at the surface), according to the initial compositions in siRNA and HSA (10/62, 40/62 and 10/206 $\mu \mathrm{g}$ of siRNA/HSA mg ${ }^{-1} \mathrm{MS}$ ), confirming that the RNPs resulting from a lower amount of siRNA/HSA adsorbed (si10-hsa62) have the smallest z-height $(11 \pm 5 \mathrm{~nm})$. In contrast, RNPs with higher ratios of siRNA/HSA (si40-hsa62 and si10-hsa206) have higher z-heights (respectively $19 \pm 9 \mathrm{~nm}$ and $24 \pm 5 \mathrm{~nm}$ ).
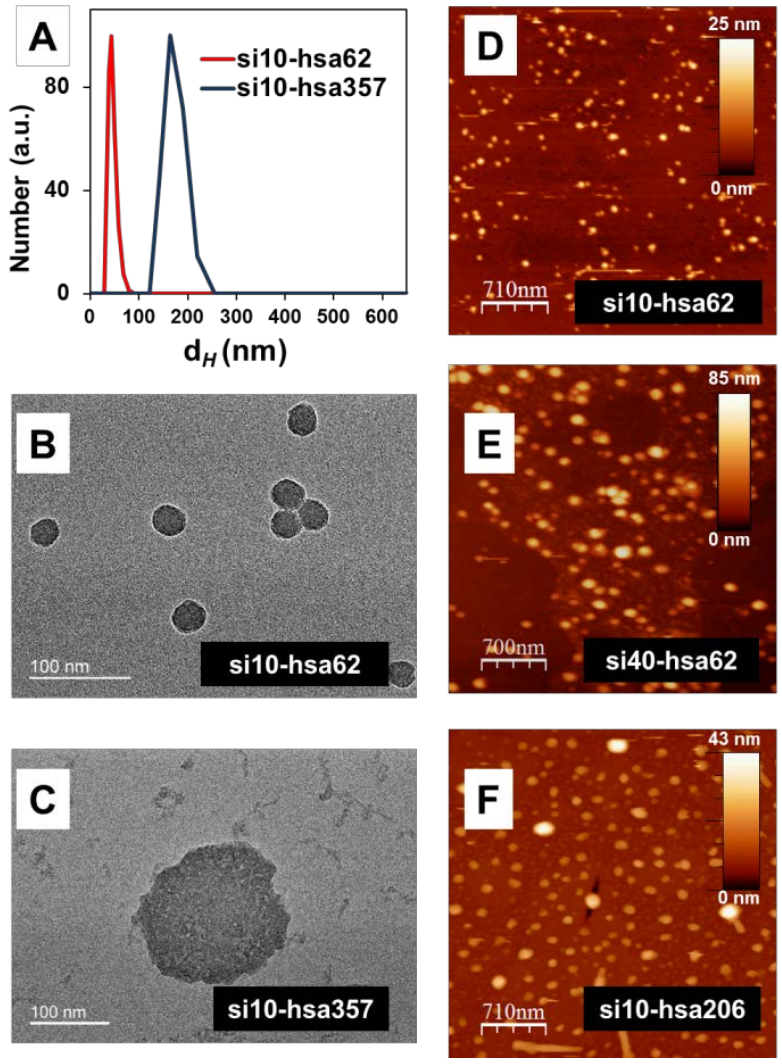

Figure 3. (A) DLS size distribution of the hydrodynamic diameter $(\mathrm{dH})$ in water measured for Gd-modified siRNA/HSA RNPs of two different formulations in siRNA and HSA : si10-hsa62 and si 10-hsa $357 \mu \mathrm{g}$ (corresponding to the siRNA and HSA quantities initially adsorbed per mg-1 of MS). TEM images (B,C) associated respectively with si10-hsa62 and si10-hsa 357 RNPs. AFM images of three different formulations in siRNA and HSA (D) si10-hsa62, (E) si40-hsa62, and (F) si10-hsa206.

The efficiency of the coupling reaction with Gd-DOTA complexes to the RNPs was investigated by elemental analysis of Gd atoms, which was performed by inductively coupled plasma-atomic emission spectroscopy (ICP-AES). In these experiments, si10-hsa62 RNPs were grafted with Gd at a ratio DOTA: $\mathrm{NH}_{2}$ 2:1 while si10hsa206 RNPs were kept at a ratio DOTA: $\mathrm{NH}_{2}$ 10:1. Respective values of $146 \pm 2$ and $780 \pm 2 \mu \mathrm{mol} . \mathrm{L}^{-1}$ in Gd element were measured in the samples si10-hsa62 and si10-hsa206 RNP dispersions (resulting from Gd-modified siRNA/HSA assemblies on MS, 2\%, $1 \mathrm{~mL}$ ). These values of Gd concentrations are in a good agreement with those found with usual contrast agents (Magnevist $^{\mathrm{TM}}$, Gadovist ${ }^{\mathrm{TM}}$ ) in clinical MRI after dilution in the blood, which is of the order of $0.5-1$ mmol. $\mathrm{L}^{-1}$. Further, the ratio in $\mathrm{Gd}$ atoms between both kinds of particles (ca. 5.), is nearly twice the ratio of HSA quantities adsorbed within the MS (ca. 3.), which shows that Gd grafting not only can be tailored with the amount of HSA adsorbed but also with the stoichiometry $\mathrm{NH}_{2}$ :DOTA:Gd. MRI properties of the RNPs were investigated in plastic tubes (1.5 and 2 $\mathrm{mL}$ volume) with a $1.5 \mathrm{~T}$ clinical MRI apparatus (Siemens) by performing $\mathrm{T}_{1}$-weighted MRI images. The results showed that both RNP dispersions displayed MRI contrast enhancements compared to a saline buffer, as shown by the vertical MRI section images and signal values measured in the tubes (Figure 4A). Gd-modified HSA RNPs formed in the absence of siRNA (resulting from Gd-modified HSA assembly on MS, 5\%, $1 \mathrm{~mL}$, at a ratio DOTA: $\mathrm{NH}_{2}$ of 2:1 for 
Gd grafting) and with high HSA adsorption rate (at $2 \mathrm{mg} \mathrm{mL}^{-1}$ in solution, with expected adsorbed amount of $261 \mu \mathrm{g}$ of $\mathrm{HSA} \mathrm{mg}^{-1}$ MS) displayed also MRI contrast enhancement. These RNPs (hsa261) were successively diluted in cascade (by 2) and imaged in transverse sections (Figure 4B). Each image is associated with a MRI signal obtained via the MRI apparatus. These MRI results indicate that these RNPs could be, for instance, well adapted to visualize targeted biological tissues in conditions where the particles can be accumulated locally.

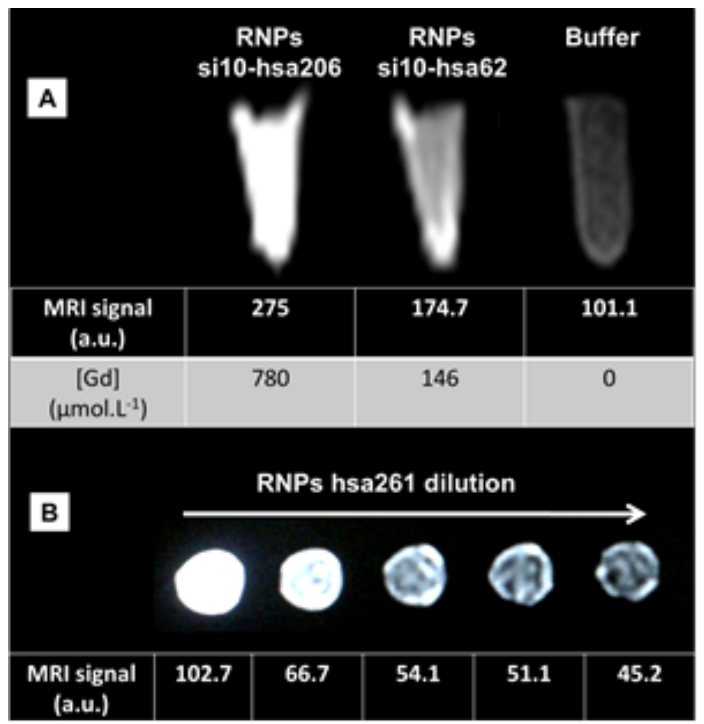

Figure 4. (A) $T_{1}$-weighted vertical section MRI images of Gdmodified siRNA/HSA RNP dispersions in Milli-Q water with low and high coverages in HSA (samples si10-hsa62 and si10-hsa206) compared to saline buffer as a control sample. (B) $\mathrm{T}_{1}$-weighted transversal section MRI images of Gd-modified HSA RNPs without siRNA adsorbed (hsa261 RNPs) at various dilutions in cascade by 2 . MRI images and signal values were acquired with a $1.5 \mathrm{~T}$ MRI (Siemens) clinical apparatus. Elemental analysis in Gd element was performed by ICP-AES analysis.

The potential of the RNPs for gene silencing was investigated with A549luc lung carcinoma cell lines stably transfected with pGL3 gene, expressing the firefly luciferase. Inhibition of the GL3 gene by RNPs bearing specifically designed siRNA: siLuc (custom siRNA, Dharmacon) in A549luc cells was monitored by bioluminescence. A549luc cells were incubated with the same amount of RNP dispersions ( $8 \mu \mathrm{L} /$ well resulting from siRNA/HSA assemblies on MS, 2\%) in 48-well plates bearing increasing amounts of siRNA per particle (7, 14, 21, 28, 35, $56 \mu$ g. $\left.\mathrm{mg}^{-1} \mathrm{MS}\right)$. After $72 \mathrm{~h}$ incubation time, cells were lysed and the luciferase activity was quantified and compared to untreated cells as control experiments. The luciferase expression level was measured as relative light unit (RLU) normalized in protein quantity. RNPs with Gd (stoichiometry DOTA: $\mathrm{NH}_{2}$ 1:1) incubated at 170, 340, $680 \mathrm{pmol} /$ well and RNPs without Gd incubated at 85, 255, $425 \mathrm{pmol} /$ well were investigated. Results showed that after $72 \mathrm{~h}$ incubation, siRNA delivery resulted in a high inhibition of the gene reporter expression in A549luc cells, with $80 \%$ of inhibition with non-Gd-grafted RNPs, and up to $50 \%$ for Gd-grafted RNPs compared with untreated cells (Figure 5A and $5 \mathrm{~B}$, respectively). The difference in gene expression might be explained by a difference in capability of the RNPs to release siRNA due to the molecular moiety involved in the Gd grafting itself. Due to the cyclic plan structure of the DOTA ligands, the DOTA-Gd grafted molecules might increase the density of the nanoparticle at the NPs surface region and thus promote a steric hindrance hampering siRNA release in the cytoplasm. Control experiments for both cases, with and without Gd grafting, showed that incubation of the cells with RNPs bearing a non-targeting gene siRNA: siCTRL (siGENOME non-targeting siRNA \#1, ref. D-001210-01-05, Dharmacon) respectively at 680 and $425 \mathrm{pmol} /$ well had no significant effect on Luc gene expression knockdown (ca. 10\% of non-specific inhibition), confirming the high specificity of the gene silencing. Further, experiments of cell viability performed with the alamarBlue test showed cell viability values ranging from $80-100 \%$, indicating no sign of cell toxicity (Figure S4). We also performed gene expression knockdown experiments by increasing the added volumes of RNPs $(1,2,4,8 \mu \mathrm{L} /$ well=15, 30, 60, $120 \mathrm{pmol} /$ well $)$ at a fixed amount of siRNA per particle $\left(10 \mu \mathrm{g}\right.$ siRNA.mg ${ }^{-1}$ MS initially incubated). The results obtained showed a dose-dependent gene silencing up to $45 \%$ associated with excellent cell viability close to $100 \%$ (Figure S5). Cell internalization of Gd-modified RNPs (si20hsa62) was visualized by confocal laser scanning microscopy (CLSM) imaging. The RNPs were tagged in green fluorescence with HSA $^{\text {FITC }}$ whereas the cytoplasm was stained in red (PKH-26 stain). Compiled CLSM images showed a homogenous distribution of the RNPs located within the cells close to the nucleus (Figure 5C), confirming their intracellular delivery. These results, combined with those of gene silencing shown above, suggest that the siRNA is delivered within the cytoplasm. Details about the cellular fate of the RNPs such as the mechanisms of their endocytosis or their endosome escape are, however, out of the scope of this communication, which emphasizes the properties of these proteinbased theranostic particles. The cell endocytosis investigation will be further addressed in detail in a future study.

\section{Conclusions}

In this work, we have designed a novel class of multifunctional protein-based nanoparticles, denoted Gd-modified siRNA/HSA RNPs, which simultaneously deliver siRNA to cells and display MRI properties. The efficacy of such RNPs in gene silencing was demonstrated with a capacity of gene expression knockdown more than $50 \%$ and a MRI contrast enhancement that would be suitable for visualization for targeted tumors. These innovative particles were assembled via the non-covalent binding with IBAM grafts, which constitute a versatile method to design novel biomacromolecular nanodevices. This method presents the advantage to avoid a further covalent cross-linking that may be time-consuming and potentially toxic. Furthermore, we expect that this process can be translated for the nanoparticle synthesis made of a range of biopolymers having relevant properties for theranostics application. . 

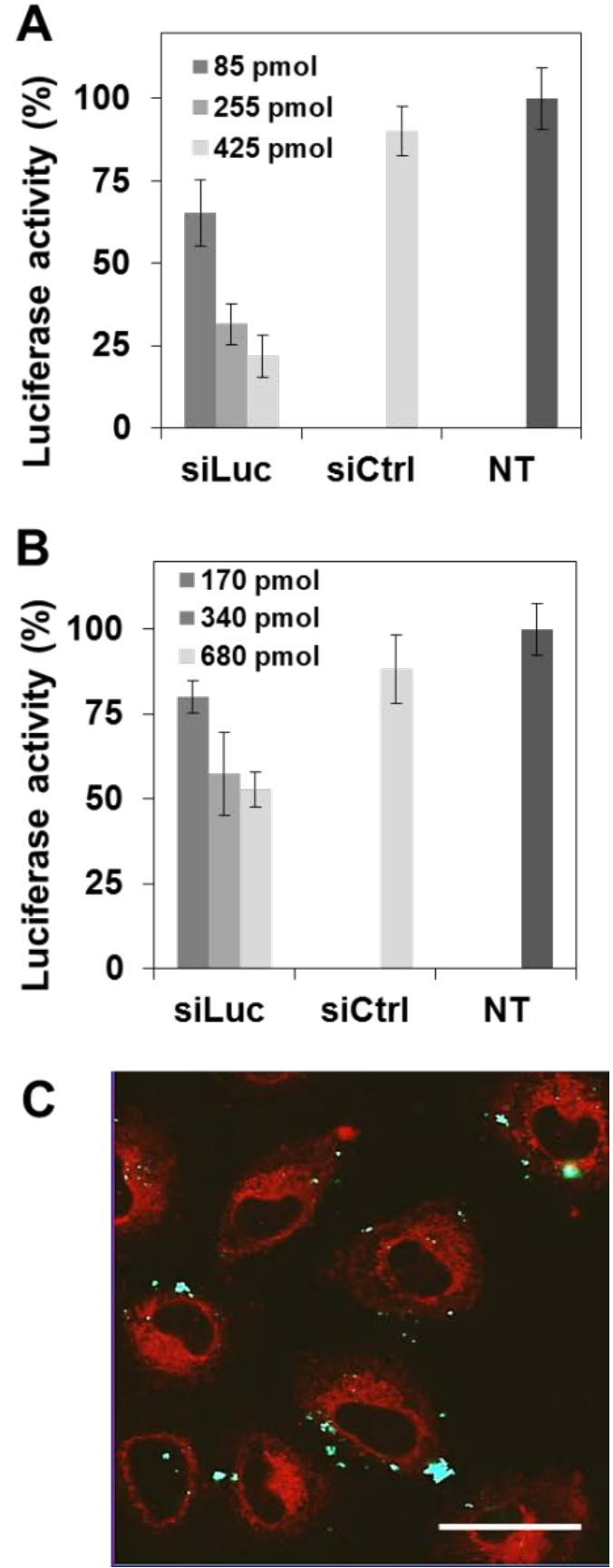

Figure 5. Biological in vitro tests. Gene silencing effect of the siRNA/HSA RNPs by measuring the luciferase activity of A549luc cells incubated with the same amount of RNP dispersions (8 $\mu \mathrm{L} /$ well) bearing increasing amounts of siRNA per particle (A) without grafted Gd (85, 255, $425 \mathrm{pmol} /$ well) and (B) with grafted Gd (170, 340, $680 \mathrm{pmol} /$ well). (C) CLSM images acquired in two fluorescence channels (green and red) showing the cell uptake of Gd-modified siRNA/HSA ${ }^{\text {FITC }}$ (si20-hsa62) RNPs (labelled in green) by $\mathrm{A} 549$ cells stained in red (cell membrane staining).

\section{Acknowledgements}

We gratefully thank Franck Blindauer for MRI imaging, Anne Boos and Pascale Ronot for ICP-AES analysis. This work was financially supported by IHU Strasbourg and “Association de Recherche contre le Cancer" (ARC, project NanoISI), LabEx HEPSYS (ANR-10LAB-28), France. F.C. acknowledges the Australian Research Council for the support under the Australian Laureate Fellowship (120100030, F.C.) and Super Science Fellowship (FS110200025, F.C.) schemes.

\section{Notes and references}

${ }^{a}$ Institut de Physique et Chimie des Matériaux de Strasbourg (IPCMS), UMR 7504, CNRS, Université de Strasbourg, 23, rue du Loess, BP 43, 67034 Strasbourg Cedex 2, France.

E-mail : damien.mertz@ipcms.unistra.fr

${ }^{b}$ Institut National de la Santé et de la Recherche Médicale, INSERM Unité 1121, 11 rue Humann, 67085, Strasbourg, France,

c Institut Hospitalo-Universitaire de Strasbourg, 1 place de l'Hôpital, 67091, Strasbourg, France.

${ }^{d}$ Faculté de chirurgie dentaire, Université de Strasbourg, 67085 Strasbourg, France.

${ }^{e}$ Department of Chemical and Biomolecular Engineering, The University of Melbourne, Parkville, Victoria 3010, Australia.

${ }^{\mathrm{f}}$ Institut National de la Santé et de la Recherche Médicale, INSERM Unité 1110, 3 rue Koeberlé, 67000 Strasbourg, France.

$\dagger$ Electronic Supplementary Information (ESI) available: [experimental details and supporting Fig. S1-S4]. See DOI: 10.1039/c000000x/

1 a) S. M. Janib, A. S. Moses, J. A. MacKay, Adv. Drug Delivery Rev. 2010, 62, 1052; b) J. Kim, Y. Piao, T. Hyeon, Chem. Soc. Rev. 2009, 38, 372; c) D.-E. Lee, H. Koo, I.-C. Sun, J. H. Ryu, K. Kim, I. C. Kwon, Chem. Soc. Rev. 2012, 41, 2656; d) N. Sanvicens, M. P. Marco, Trends Biotechnol. 2008, 26, 425.

2 a) T. Lammers, S. Aime, W. E. Hennink, G. Storm, F. Kiessling, Acc. Chem. Res. 2011, 44, 1029; b) B. Sumer, J. Gao, Nanomedicine 2008, 3, 137.

3 a) S. I. Pai, Y. Y. Lin, B. Macaes, A. Meneshian, C. F. Hung, T. C. Wu, Gene Ther. 2005, 13, 464; b) D. H. Kim, J. J. Rossi, Nat. Rev. Genet. 2007, 8, 173; c) G. J. Hannon, Nature 2002, 418, 244.

4 M. E. Davis, Mol. Pharm. 2009, 6, 659-668.

5 Petros et al. Nature reviews Drug discovery 2010...

6 a) A. L. Becker, A. P. R. Johnston, F. Caruso, Small 2010, 6, 1836; b) B. Ozpolat, A. K. Sood, G. Lopez-Berestein, J. Intern. Med. 2009, 267, 44; c) D. Reischl, A. Zimmer, Nanomed. Nanotechnol. Biol. Med. 2009, 5, 8.

7 a) A. P. R. Johnston, C. Cortez, A. S. Angelatos, F. Caruso, Curr. Opin. Colloid Interface Sci. 2006, 11, 203; b) Cui, J.; van Koeverden, M. P.; Müllner, M.; Kempe, K.; Caruso, F. Adv. Colloid Interface Sci. 2014, DOI: 10.1016/j.cis.2013.1010.1012.

8 A. L. Becker, N. I. Orlotti, M. Folini, F. Cavalieri, A. N. Zelikin, A. P. R. Johnston, N. Zaffaroni, F. Caruso, ACS Nano 2011, 5, 1335.

9 J.-H. Lee, K. Lee, S. H. Moon, Y. Lee, T. G. Park, J. Cheon, Angew. Chem. 2009, 121, 4238.

10 F. Kratz, J. Control. Release 2008, 132, 171.

11 a) D. Mertz, J. Cui, Y. Yan, G. Devlin, C. Chaubaroux, A. Dochter, R. Alles, P. Lavalle, J. C. Voegel, A. Blencowe, P. Auffinger, F. Caruso, ACS Nano 2012, 6, 7584; b) D. Mertz, P. Tan, Y. Wang, T. K. Goh, A. Blencowe, F. Caruso, Adv. Mater. 2011, 23, 5668; c) D. Mertz, H. Wu, J. S. Wong, J. Cui, P. Tan, R. Alles, F. Caruso, J. Mater. Chem. 2012, 22, 21434.

12 a) J. Cui, Y. Yan, Y. Wang, F. Caruso, Adv. Funct. Mater. 2012, 22, 4718; b) Y. J. Wang, F. Caruso, Adv. Mater. 2006, 18, 795; c) J. Cui, 
R. De Rose, J. P. Best, A. P. R. Johnston, S. Alcantara, K. Liang, G. K. Such, S. J. Kent, F. Caruso, Adv. Mater. 2013, 25, 3468.

13 J. G. Wang, H. J. Zhou, P. C. Sun, D. T. Ding, T. H. Chen, Chem. Mater. 2010, 22, 3829.

\section{The table of contents entry}

HSA-based nanoparticles bearing silencing RNA and gadolinium complexes are obtained via templated assembly on isobutyramide modified-mesoporous silica. These protein particles provide simultaneous magnetic resonance imaging contrast and cellular gene silencing.

\section{TOWARDS ALBUMIN-BASED NANOTHERANOSTICS}

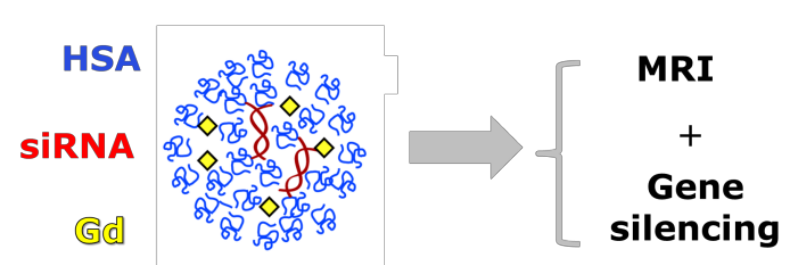




\section{University Library}

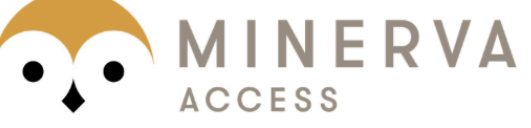

A gateway to Melbourne's research publications

Minerva Access is the Institutional Repository of The University of Melbourne

Author/s:

Mertz, D;Affolter-Zbaraszczuk, C;Barthes, J;Cui, J;Caruso, F;Baumert, TF;Voegel, J-C;Ogier, J;Meyer, F

Title:

Templated assembly of albumin-based nanoparticles for simultaneous gene silencing and magnetic resonance imaging

Date:

2014-01-01

Citation:

Mertz, D., Affolter-Zbaraszczuk, C., Barthes, J., Cui, J., Caruso, F., Baumert, T. F., Voegel, J. -C., Ogier, J. \& Meyer, F. (2014). Templated assembly of albumin-based nanoparticles for simultaneous gene silencing and magnetic resonance imaging. NANOSCALE, 6 (20), pp.11676-11680. https://doi.org/10.1039/c4nr02623c.

Persistent Link:

http://hdl.handle.net/11343/123258 